wie sie durch Einführung eines ganz neuen neutralen Meridians allen denjenigen Ländern zugemuthet werden würden, die den Meridian von Greenwich in allen bezüglichen Einrichtungen schon angenommen haben.

Auf jene Frage kann aber die Antwort nach dem Obigen nur lauten: „Es giebt keinerlei zwingende Gründe bei dieser völlig neuen Einführung eine andere Grundlage zu wählen, als den astronomischen Tag zu Greenwich, den astronomischen und nautischen Universal-Tag. - Vielmehr genügt die Wahl dieser Grundlage auch den tieferen sachlichen Bedürfnissen, für welche ausserhalb der Astronomie und der Nautik die Einführung einer vom Orte unabhängigen, unzweideutigen und allgemein gültigen Zeitangabe noch erforderlich ist, auf einfachere und gunstigere weise als die Wahl eines Universal-Tages, welcher nichts Anderes als die Genugthuung darbietet, dass er sich dem bürgerlichen Tage Englands vollständig anschliesst. \&

$\mathrm{Da}$ in diesen Blättern wesentlich die Astronomie zu vertreten ist, und es der Nautik überlassen werden kann, sich selbst gegen die Einführung des bürgerlichen Greenwicher Tages $\mathrm{zu}$ wehren, dürften einige Worte genügen, um es, angesichts des obigen Sachverhaltes, zunächst von Herrn Professor Tietjen's und meiner Seite zu rechtfertigen, dass der neue Universal-Tag in astronomischen Jahrbuche nicht angenommen wird.

Berlin $\mathbf{1 8 8 4}$ Jan. I I.
Im Jahrbuch war es beabsichtigt, schon vom neuesten Jahrgange $a b$ für alle diejenigen Zeitangaben, welche eine generelle und von dem Beobachtungsbedürnisse der deutschen Meridiane nahezu unabhängige Bedeutung haben, entsprechend den Vorschlägen der Conferenz zu Rom, den Greenwicher astronomischen Tag als Universal-Tag und die Greenwicher astronomische Zeit als Universalzeit (U. T.) anzunehmen. Nachdem es inzwischen unsicher geworden ist, ob diese Grundlage demnächst eine allgemeine werden wird, soll bis auf Weiteres die bisherige Datirung beibehalten werden.

Keinesfalls wird im Jahrbuch eine so schwerwiegende Discontinuität, wie sie mit einer Verlegung des astronomischen Tagesanfanges verbunden sein würde, ohne gründliche und langjährige Erwägungen und ohne die Zustimmung von Seiten aller competenten Fachmänner jemals ins Werk gesetzt werden. Die diesseitigen Ueberzeugungen aber widerstreben, wie ich zugleich im Namen der Herren Geheim. rath Auwers und Professor Tietjen erklären kann, aufs Entschiedenste dieser grossen Störung der astronomischen Continuität, mit welcher nach keiner anderen Seite hin Vortheile und auch für die astronomische Arbeit überwiegende Nachtheile und Erschwernisse verbunden sein wiirden.

\title{
Zusätze und Bemerkungen zu Laplace's Untersuchung über die Gestalt der Saturnsringe.
}

\author{
Von Frau Sophie Kowalewsky in Stockholm.
}

Auf meine Bitte hat mir die Verfasserin erlaubt, das beifolgende Manuscript der Redaction der A. N. zu überreichen. Die Abhandlung ist zwar schon vor mehreren Jahren verfasst, bis jetzt jedoch nicht gedruckt worden, und so meine ich, dass die Veröffentlichung der hochinteressanten Untersuchung den Lesern der A. N. nur angenehm sein kann.

\section{Hugo Gyldén.}

Laplace hat in dem Abschnitte der Mécanique céleste, welcher sich mit der Gestalt der Himmelskörper beschäftigt, auch die Gestalt der Saturnsringe theoretisch zu bestimmen unternommen. Die Grundlage seiner Untersuchungen bildet das folgende Problem:

Ein mit homogener Masse angefullter Ring, entstanden durch die Umdrehung einer Ellipse um eine sie nicht schneidende, aber in ihrer Ebene liegende und einer ihrer Hauptaxen parallelen Geraden, rotire mit gleichförmiger Geschwindigkeit um diese Gerade (die Axe des Ringes). Die Oberfläche des Ringes sei mit einer unendlich dünnen Schicht einer homogenen Fliussigkeit bedeckt, welche der Anziehung des Ringes und ausserdem der Anziehung eines Central-Körpers, dessen Schwerpunkt mit dem Mittelpunkt des Ringes zusammenfällt, unterworfen ist. Es fragt sich, ob die Elemente des Ringes (die Halbaxen der erzeugenden Ellipse und der Abstand des Mittelpunkts derselben von der Rotationsaxe) und seine Undrehungsgeschwindigkeit so bestimmt werden können, dass die Flüssigkeit im Gleich- gewichtszustande (in Beziehung auf die Oberfläche des Ringes) beharren könne, wozu bekanntlich erforderlich und hinreichend ist, dass die folgende Gleichung bestehe, in der

$n$ die Rotationsgeschwindigkeit des Ringes,

$V$ das Potential desselben in Beziehung auf einen unbestimmten Punkt der Oberfläche desselben,

$\rho_{1}$ den Abstand dieses Punktes von der Rotationsaxe,

$z_{1}$ dessen Abstand von der Aequatorialebene,

$M$ die in ihrem Schwerpunkt concentrirt gedachte Masse des Centralkörpers,

$C$ eine Constante bedeutet:

$$
V+\frac{M}{\sqrt{{Q_{1}^{2}}^{2}+z_{1}^{2}}}+1 / 2 n^{2} \varrho_{1}{ }^{2}-C=0
$$

Laplace untersucht aber die Aufgabe nur unter der Annahme, dass die Oeffnung des Ringes (d. h. der Abstand seines Mittelpunktes von dem Mittelpunkte der erzeugenden 
Ellipse) sehr gross sei im Verhältniss zu den Halbaxen der letzteren. Da bei dieser Annahme ein Theilchen der Oberfläche des Ringes von den entfernten Theilen desselben nur eine sehr schwache Anziehung erfährt, so betrachtet Laplace bei der Berechnung von $V$ den als wirksam angenommenen Theil des Ringes als einen unendlichen elliptischen Cylinder, und kommt so zu folgendem Resultate: Wenn man das Verhältniss der grossen Axe der erzeugenden Ellipse des Ringes zu dem Abstande des Mittelpunkts desselben von der Rotationsaxe als eine kleine Grösse erster Ordnung ansieht, so ist es möglich, bei gegebener Masse des Centralkörpers, die Rotationsgeschwindigkeit und das Verhältniss beider Axen jener Ellipse so zu bestimmen, dass die vorstehende Gleichung annäherungsweise und zwar bis auf eine kleine Grösse dritter Ordnung (exclusive) befriedigt wird.

Aber die Annahme von Laplace, dass bei der Bestimmung von $V$ dem Ringe ein Cylinder substituirt werden könne, ist insofern unbefriedigend als sich nicht ohne Weiteres beurtheilen lässt, ob der dabei begangene Fehler wirklich von der angegebenen Ordnung ist. Ausserdem lässt die Laplace'sche Behandlung der Aufgabe nicht erkennen, ob und wie man durch eine kleinere Aenderung des elliptischen Querschnitts des Ringes bewirken könne, dass der Ausdruck auf der linken Seite der Gleichung (I) entweder wirklich Null sei oder doch dieser Grenze beliebig nahe gebracht werden könne.

Aus diesen Gründen habe ich die in Rede stehende Aufgabe auf eine andere Weise $z u$ behandeln versucht.

Ich nehme an, dass die Linie, welche die erzeugende Figur des Ringes begrenzt und die ich im Folgenrlen mit $L$ bezeichnen will, wenig von einer Ellipse abweiche, gleich dieser eine, in ihrer Verlängerung die Rotationsaxe des Ringes unter rechtem Winkel schneidende Symmetrieaxe besitze und überdies so gestaltet sei, dass jede der Symmetrieaxe parallele gerade Iinie sie nur in zwei Punkten trifft.

Wenn ich dann ein orthogonales Axensystem annehme, dessen $Z$-Axe die Rotationsaxe des Ringes ist, während die $X Y$-Ebene durch die genannte Symmetrielinie geht, so kann ich die Oberfäche des Ringes, das Mittel zwischen dem kleinsten und grössten Abstande derselben von der $Z$-Axe als Längeneinheit annehmend, durch zwei Gleichungen von der Form

$$
\begin{aligned}
\sqrt{x^{2}+y^{2}} & =1-a \cos t \\
z & =a\left(\beta \sin t+\beta_{1} \sin 2 t+\beta_{2} \sin 3 t+\cdots\right)
\end{aligned}
$$

darstellen, wo $t$ eine reelle Veränderliche bedeutet, die alle Werthe von $\circ$ bis $2 \pi \mathrm{zu}$ durchlaufen hat,

$$
a, \beta, \beta_{1}, \beta_{2} \ldots
$$

aber Constanten bedeuten.

Unter Beibehaltung der Laplace'schen Annahme, dass jede Sehne von $L$ sebr klein sei im Verhältniss zum mittleren Abstande dieser Linie von der $Z$-Axe, ist in diesen Formeln $a$ als ein sehr kleiner Bruch vorauszusetzen; damit aber $L$ wenig von einer Ellipse abweiche, müssen nicht nur $\beta_{1}, \beta_{2} \ldots$ sämmtlich kleine Grössen, sondern auch die Summe ihrer absoluten Beträge klein im Verhältniss zu $\beta$ sein.

Dieses vorausgesetzt, sei do irgend ein unendlich kleines Element der Ringoberfläche, $P$ ein Punkt desselben, $P_{1}$ ein bestimmter Punkt der Oberfläche, für den das Potential $V$ berechnet werden soll, $\boldsymbol{\theta}$ der Winkel, welchen die in $P$ auf der Aussenseite der Fläche errichtete Normale mit der Geraden $P P_{1}$ bildet; so ist, nach Gauss (Gesammelte Werke, Bd. V, Handschriftliche Bemerkung zu der Abhandlung $\gg$ Theoria attractionis corporum sphaeroidicorum ellipticorum homogeneorum\&, S. 286)

$$
V=-1 / 2 \pi \cos \theta \mathrm{d} \sigma
$$

Sind $x, y, z$ die Coordinaten von $P, x_{1}, y_{1}, z_{1}$ die von $P_{1}, r$ der Abstand dieser beiden Punkte und $\xi, \eta, \zeta$ die Cosinus der Winkel, welche die genannte Normale mit den positiven Richtungen der Coordinatenaxen bildet, so hat man

$$
\cos \theta=\frac{x_{1}-x}{r} \xi+\frac{y_{1}-y}{r} \eta+\frac{z_{1}-z}{r} \zeta
$$

Wird ferner

$$
x=\sqrt{x^{2}+y^{2}} \cdot \cos \psi, \quad y=\sqrt{x^{2}+y^{2}} \cdot \sin \psi
$$

gesetzt, so hat man

$$
\begin{aligned}
& x=(1-a \cos t) \cos \psi \\
& y=(1-a \cos t) \sin \psi \\
& z=a \varphi(t)=a\left(\beta \sin t+\beta_{1} \sin 2 t+\cdots\right)
\end{aligned}
$$

und

$$
\begin{aligned}
& x_{1}=\left(\mathrm{I}-a \cos t_{1}\right) \cos \psi_{1} \\
& y_{1}=\left(\mathrm{I}-a \cos t_{1}\right) \sin \psi_{1} \\
& z_{1}=a \varphi\left(t_{1}\right)
\end{aligned}
$$

Setzt man also zur Abkürzung

$$
\begin{gathered}
A=4(1-a \cos t)\left(1-a \cos t_{1}\right) \\
B=a^{2}\left[\left(\cos t-\cos t_{1}\right)^{2}+\left[\varphi(t)-\varphi\left(t_{1}\right)\right]^{2}\right] \\
C=2 a^{2}(1-a \cos t)\left[\left(\cos t-\cos t_{1}\right) \varphi^{\prime}(t)\right. \\
\left.\quad+\sin t\left[\varphi(t)-\varphi\left(t_{1}\right)\right]\right]
\end{gathered}
$$

so wird

$$
V=\frac{1}{4} \int_{0}^{2 \pi} \mathrm{d} t \int_{0}^{2 \pi} \frac{C-A a \varphi^{\prime}(t) \sin ^{21 / 2}\left(\psi-\psi_{1}\right)}{\sqrt{B+A \sin ^{21 / 2}\left(\psi-\psi_{1}\right)}} \mathrm{d} \psi
$$

oder wenn man

$$
\begin{aligned}
& \vartheta=\int^{1 / 2}\left(\psi-\psi_{1}\right) \\
& W=\int_{0} \frac{C-A a \varphi^{\prime}(t) \sin ^{2} \vartheta}{\sqrt{B+A \sin ^{2} \vartheta}} \mathrm{d} \vartheta \text { setzt }
\end{aligned}
$$




$$
V=\int_{0}^{2 \pi} W \mathrm{~d} t
$$

Das Integral $W$ forme ich um, indem ich

$$
\sqrt{A \sin ^{2} \theta+B}=\sqrt{B} \cdot s
$$

setze, wodurch sich

$$
W=\int_{\mathrm{I}}^{\frac{\mathrm{I}}{k}} \frac{C+B a \varphi^{\prime}(t)-B a \varphi^{\prime}(t) s^{2}}{\sqrt{A+B} \cdot \sqrt{s^{2}-\mathbf{I}} \cdot \sqrt{\mathrm{I}-k^{2} s^{2}}} \mathrm{~d} s
$$

ergiebt, wo

$$
k^{2}=\frac{B}{A+B}
$$

Da $k^{2}$, der Annahme nach, eine sehr kleine Grösse ist, so bedient man sich zur Berechnung von $W$ am besten der bekannten Formel (Weierstrass, "Theorie der Abel'schen Functionen Crelle's Journal B. 52, S. 75 und 80):

$$
\begin{aligned}
& \int_{1}^{\frac{1}{k}} \frac{\mathrm{d} \xi}{\sqrt{\xi^{2}-1} \sqrt{1-k^{2} \xi^{2}}}=\frac{\mathrm{I}}{2} K \log \frac{\mathrm{I} 6}{k^{2}}-\bar{K} \\
& \int_{\mathrm{I}}^{\frac{\mathrm{I}}{k}} \frac{k^{2} \xi^{2} \mathrm{~d} \xi}{\sqrt{\xi^{2}-1} \sqrt{\mathrm{I}-k^{2} \xi^{2}}}=\frac{\mathrm{I}}{2} \mathscr{F} \log \frac{\mathrm{I} 6}{k^{2}}-\overline{\mathcal{F}}
\end{aligned}
$$

wo

$$
\begin{aligned}
& K=\mathrm{I}+\left(\frac{\mathrm{I}}{2}\right)^{2} k^{2}+\left(\frac{\mathrm{I} \cdot 3}{2 \cdot 4}\right)^{2} k^{4}+\cdots \\
& \bar{K}=\left(\frac{\mathrm{I}}{2}\right)^{2} k^{2}+\frac{7}{2 \cdot 3}\left(\frac{\mathrm{I} \cdot 3}{2 \cdot 4}\right)^{2} k^{4}+\cdots \\
& \mathcal{F}=\frac{\mathrm{I}}{2} k^{2}+\frac{3}{4}\left(\frac{\mathrm{I}}{2}\right)^{2} k^{4}+\cdots \\
& \overline{\mathcal{F}}=-\mathrm{I}+\frac{\mathrm{I}}{4} k^{2}+\frac{\mathrm{I} 3}{12} \cdot \frac{3}{4}\left(\frac{\mathrm{I}}{2}\right)^{2} k^{4}+\cdots
\end{aligned}
$$

Dann ist

$$
W=W_{1} \log \frac{16}{k^{2}}+W_{2}
$$

พo

$$
\begin{aligned}
& W_{1}=\frac{\mathrm{x}}{2} \frac{C+a B \varphi^{\prime}(t)}{\sqrt{A+B}} K-\frac{\mathrm{x}}{2} \frac{a B \varphi^{\prime}(t)}{\sqrt{A+B}} \mathcal{F} \\
& W_{2}=-\frac{C+a B \varphi^{\prime}(t)}{\sqrt{A+B}} \bar{K}+\frac{a B \varphi^{\prime}(t)}{\sqrt{A+B}} \overline{\mathcal{Y}}
\end{aligned}
$$

Die Grössen $W_{1}, W_{2}$ lassen sich nach den Cosinus und Sinus von $t_{1} t_{1}$ in Reihen entwickeln, und zwar so, dass die Coefficienten Potenzreihen der Grössen $a, \beta, \beta_{1} \ldots$ werden. Diese Reihen sind schon der Kleinheit von $a$ halber sehr stark convergent.

Das Integral

$$
\int_{0}^{2 \pi} W_{2} \mathrm{~d} t
$$

lässt sich dann ohne Weiteres dadurch bestimmen, dass man das von $t$ unabhängige Glied der Reihe $W_{2}$ mit $2 \pi$ multiplicirt.

Nicht so einfach verhält es sich mit dem Integral

$$
\int_{\mathrm{o}}^{2 \pi} W_{1} \log \frac{\mathrm{I} 6}{k^{2}} \mathrm{~d} t
$$

Die Grösse $B=a^{2}\left[\left(\cos t-\cos t_{1}\right)^{2}+\left[\varphi(t)-\varphi\left(t_{1}\right)\right]^{2}\right]$ wird, als Function von $t$ betrachtet, nur gleich Null, wenn gleichzeitig $\cos t=\cos t_{1}$ und $\varphi(t)=\varphi\left(t_{1}\right)$ wird, was bei der angenommenen Gestalt der Linie $L$ nur geschehen kann, wenn $t=t_{1}+2 m \pi$ ist, wo $m$ eine ganze $Z a h l$ bedeutet; daraus folgt, dass $B$ durch $\mathrm{I}--\cos \left(t-t_{1}\right)$ theilbar ist. Man kann also setzen

$$
B=a^{2}\left[\mathbf{1}-\cos \left(t-t_{1}\right)\right] B_{1}
$$

wo $B_{1}$ nach den Cosinus und Sinus von $t, t_{1}$ entwickelbar ist. Setzt man $\beta_{1}, \beta_{2} \ldots$ sämmtlich gleich Null, so reducirt sich $B_{1}$ auf

$$
1 / 2\left(1+\beta^{2}\right)-1 / 2\left(1-\beta^{2}\right) \cos \left(t+t_{1}\right)
$$

woraus folgt, dass $B_{1}$ bei hinlänglicher Kleinheit der Grössen $\beta_{1}, \beta_{2} \ldots$ für reelle Werthe von $t, t_{1}$ nicht verschwindet. Die Coefficienten der einzelnen Glieder von $B_{1}$ sind übrigens wieder ganze Functionen von $a, \beta, \beta_{1} \ldots$

Setzt man nun

$$
B_{1}=\left[1 / 2\left(1+\beta^{2}\right)-1 / 2\left(1-\beta^{2}\right) \cos \left(t+t_{1}\right)\right]\left(\mathbf{1}+B_{3}\right)
$$

so ist

$$
\begin{gathered}
\log B=2 \log a+\log \left[\mathbf{1}-\cos \left(t-t_{1}\right)\right] \\
+\log \left[1 / 2\left(\mathbf{I}+\beta^{2}\right)-1 / 2\left(\mathbf{1}-\beta^{2}\right) \cos \left(t+t_{1}\right)\right]+\log \left(\mathbf{r}+B_{2}\right)
\end{gathered}
$$

und es setzt sich $W_{1} \log \frac{\mathbf{r} 6}{k^{2}}$ aus folgenden fünf Theilen $z u-$ sammen :

$$
\begin{aligned}
& W_{1} \log \mathrm{r} 6(A+B) \\
- & 2 W_{1} \log a \\
- & W_{1} \log \left[1 / 2\left(1+\beta^{2}\right)-1 / 2\left(1-\beta^{2}\right) \cos \left(t+t_{1}\right)\right] \\
- & W_{1} \log \left[1-\cos \left(t-t_{1}\right)\right] \\
- & W_{1} \log \left(1+B_{2}\right)
\end{aligned}
$$

Die drei ersten Theile kann man in Reihen von derselben Form wie $W_{2}$ entwickeln und dann die ihnen entsprechenden Theile des Integrals 


$$
\int_{0}^{2 \pi} W_{1} \log \frac{16}{k^{2}} \mathrm{dt}
$$

unmittelbar bestimmen. Der vierte Theil lässt sich nur für die zwischen $0 \ldots 2 \pi$ liegenden Werthe von $t-t_{1}$ in eine Reihe von der angegebenen Gestalt entwickeln; nach dem Fourier'schen Satze aber ist dann noch immer das von $t$ unabhängige Glied, wenn es mit $2 \pi$ multiplicirt wird, der Werth des Integrals

$$
-\int_{0}^{2 \pi} W_{1} \log \left[\mathrm{I}-\cos \left(t-t_{1}\right)\right] \mathrm{d} t
$$

Zur Berechnung des Integrals

$$
\int_{0}^{2 \pi} W_{1} \log \left(1+B_{2}\right) d t
$$

hat man zunächst $\log \left(1+B_{2}\right)$ nach Potenzen von $B_{2}$ in eine Reihe $z u$ entwickeln, welche wegen der angenommenen Kleinheit der Summe der absoluten Beträge von $\beta_{1}, \beta_{2} \ldots$ stark convergent ist; worauf man dann die Integration ebenfalls nach bekannten Formeln ausfuhren kann.

Auf diese Weise erhält man $V$ durch eine vollständig bestimmte und stark convergirende Reihe von der Form dargestellt.

$$
V_{0}+V_{1} \cos t_{1}+V_{2} \cos 2 t_{1}+\cdots
$$

Die Coefficienten dieser Reihe sind in Beziehung auf $a, \log a, \log (\mathrm{I}+\beta), \beta_{1}, \beta_{2} \ldots$ ganze Functionen (von unendlich vielen Gliedern), deren Coefficienten gebrochene, rationale Functionen von $\beta$ sind: 'dabei ist zu bemerken, dass $\log a$ nur multiplicirt mit einer Potenz von $a$ vorkommt.

Die in Gleichung ( $\mathrm{I}$ ) vorkommende Grösse

$$
\frac{M}{\sqrt{{\rho_{1}{ }^{2}+z_{1}^{2}}^{2}}}=\frac{M}{\sqrt{\left(\mathrm{I}-a \cos t_{1}\right)^{2}+a^{2} \varphi^{2}\left(t_{1}\right)}}
$$

lässt sich ebenfalls in eine stark convergirende Reihe von der Form

$$
m_{0}+m_{1} \cos t_{1}+m_{2} \cos 2 t_{1}+\cdots
$$

entwickeln, wo die Coefficienten $m_{0}, m_{1} \ldots$ ganze Functionen (mit unendlich vielen Gliedern) von $\alpha, \beta, \beta_{1} \ldots$ sind.

Endlich ist

$\varphi_{1}^{2}=\left(1-a \cos t_{1}\right)^{2}=1+1 / 2 a^{2}-2 a \cos t_{1}+1 / 2 a^{2} \cos 2 t_{1}$

Wenn also bei einem Ringe von der angegebenen Gestalt die ihn bedeckende Flüssigkeit während der Rotation des Körpers im Gleichgewichts.Zustande soll beharren können, so muss es möglich sein die Grössen

$$
n, C, \beta, \beta_{1}, \beta_{2} \ldots
$$

so $\mathrm{zu}$ bestimmen, dass

$$
\text { (I I). } \begin{array}{ll}
V_{1}+m_{1}-n^{2} a & =0 \\
V_{2}+m_{2}+1 / 4 n^{2} a^{2} & =0
\end{array}
$$$$
V_{0}+m_{0}+1 / 2 n^{2}\left(1+1 / 2 a^{2}\right)+C=0
$$

und für jeden positiven Werth von $\nu$

$$
V_{y+2}+m m_{y-1-2}=0
$$

wird.

Diese (Fleichungen werden aber sämmtlich nur zu befriedigen sein, wenn die Reihe der Grössen $\beta, \beta_{1}, \beta_{2} \ldots$ eine unendliche ist, da aber dann in jede einzelne Gleichung auch unendlich viele dieser Grössen eingehen, so ist es nicht möglich, dieselben direct zu bestimmen. Man könnte aber, wenn die Durchführung der Rechnung nicht auf unüberwindliche Schwierigkeiten führte, folgendes Verfahren versuchen. Man nehme

$$
\varphi(t)=\beta^{(\mu)} \sin t+\beta_{1}^{(\mu)} \sin 2 t+\cdots \cdot \beta_{\mu}^{(\mu)} \sin (\mu+x) t
$$

an, berechne die Coefficienten $V_{\lambda}, m_{\lambda}$ und bestimme

$$
n^{(\mu)}, C^{(\mu)}, \beta^{(\mu)}, \beta_{1}^{(\mu)} \cdot \cdots \beta_{\mu}^{(\mu)}
$$

so, dass von den Gleichungen (I I, I 2) die $(\mu+3)$ ersten befriedigt werden. Wenn sich dann zeigen liesse, dass diese Grössen für $\mu=\infty$ sich bestimmten endlichen Grenzen näherten, und zwar so, dass die Summe der absoluten Beträge der Grenzwerthe von $\beta_{1}, \beta_{2} \ldots$ als klein im Verhältniss zum Grenzwerth von $\beta$ sich herausstellte, so würde man schliessen können, dass die Gleichungen (I I, I 2) dadurch zu befriedigen seien, dass man für jede der Grössen

den Grenzwerth

$$
n^{(\mu)}, C^{(\mu)}, \beta^{(\mu)}, \beta_{1}^{(\mu)} . .
$$

setzte.

$$
n, C, \beta, \beta_{1} \ldots
$$

Dieses Raisonnement soll aber nur dazu dienen, um die Möglichkeit einer Berechnung dieser Grössen anschaulich $z u$ machen. Eine beliebig weit $z u$ treibende angenäherte Bestimmung derselben kann man aber auf folgende Weise erhalten. Man mache,

$$
\varphi=\beta \sin t+\beta_{1} \sin 2 t+\cdots \cdot \beta_{\mu} \sin (\mu+1) t
$$

setzend, vorläufig nur die Annahme, dass die Summe der absoluten Beträge $\operatorname{der} \beta_{1} \ldots \beta_{\mu}$ eine kleine Grösse von derselben Ordnung wie $a$ sei, und lasse bei der vorhin auseinandergesetzten Entwickelung von $V_{\lambda}, m_{\lambda}$ alle Glieder von höherer als von der $(\mu+2)^{\text {ton }}$ Ordnung weg, wobei man $a^{m} \log a$ als eine kleine Grösse von höherer als der $(m-r)^{\text {ton }}$ Ordnung anzusehen hat.

Wenn man dann von den Gleichungen (I I, I 2) die $(\mu+3)$ ersten genauer untersucht, so erkennt-man, dass die sich aus ihnen ergebenden Werthe von $\beta_{1}, \beta_{2} \ldots \beta_{\mu}$ kleine Grössen bezüglich von der $I^{\text {ten }}, 2^{\text {ten }} \ldots \mu^{\text {ten }}$ Ordnung werden; man kann also setzen 


$$
\begin{aligned}
& \beta_{1}=a \gamma_{1} \\
& \beta_{2}=a^{2} \gamma_{2} \\
& \cdot \cdot \cdot \cdot \\
& \beta_{\mu}=a^{\mu} \gamma_{\mu}
\end{aligned}
$$

in der Art, dass man aus den genannten Gleichungen $\gamma_{1} \ldots \gamma_{\mu}$ als endliche Grössen erhält, und zwar, nachdem $\beta_{0}$ bestimmt ist, eine jede eindeutig; zugleich ergiebt sich, dass für die so bestimmten Grössen $\beta_{1} \ldots \beta_{\mu}$ die Ausdrücke auf der linken Seite aller übrigen Gleichungen (I I, I 2 ) kleine Grössen von höherer als der $(\boldsymbol{u}+2)^{\text {ten }}$ Ordnung werden. So ergiebt sich das Resultat:

Es ist möglich den Querschnitt des Ringes so zu bestimmen, dass der Ausdruck

$$
V+\frac{M}{\sqrt{\varrho^{2}+z^{2}}}+1 / 2 n^{2} \varrho^{2},
$$

der auf der Oberfläche des Ringes einen unveränderlichen Werth haben sollte, von einer Constanten überall nur um eine kleine Grösse von höherer als der $(\mu+2)^{\text {ten }}$ Ordnung - die Dicke des Ringes $2 a$ als eine kleine Grösse erster Ordnung angenommen - verschieden ist.

$\mathrm{Da}(\mu+2)$ beliebig gross angenommen werden kann, so darf man schliessen, dass eine Gestalt des Querschnitts des Ringes, bei welcher der vorstehende Ausdruck auf der Oberfläche des Ringes constant ist, wirklich existirt.

Ich habe diese - immerhin noch sehr weitläufige Rechnung für $\boldsymbol{u}=\mathrm{I}$ vollständig durchgeführt, hauptsächlich um zu prüfen, bis wie weit das Laplace'sche Resultat angenähert richtig sei, und um die Abweichung des Querschnittes des Ringes von der elliptischen Gestalt wenigstens in ihrem beträchtlichsten Theile zu ermitteln.

Den Querschnitt des Ringes noch genauer zu bestimmen hat mich übrigens, ausser der Schwierigkeit der Rechnung, der Umstand abgehalten, dass nach den Untersuchungen von Maxwell (On the Stability of the motion of Saturn's Rings, Cambridge r 859) es zweifelhaft geworden ist, ob die Ansicht Laplace's von der Constitution der Saturnsringe uberhaupt haltbar ist.

Setzt man die Masse des Centralkörpers gleich Null, so ist die Oberfläche des Ringes identisch mit der, welche er haben würde, wenn seine Masse aus einer homogenen Flüssigkeit bestände. Nun hat zwar die Frage, ob ein solcher flüssiger Ring von der hier betrachteten Form, wenn er sich gleichförmig um seine Axe dreht, im Gleichgewichtszustande beharren könne, ein bedeutendes theoretisches Interesse; indessen würde es, um dieselbe bejahen zu können, ausser der exacten Bestimmung der durch die Gleichung

$$
V+1 / 2 n^{2} \rho^{2}+C=0
$$

definirten Gestalt der Oberfläche des Ringes, noch einer Untersuchung in Betreff der Stabilität des Gleichgewichts bedürfen, wozu mir bis jetzt die Mittel fehlen.

Ich setze jetzt die Formeln her, welche zur Bestimmung des Querschnitts des Ringes mit einer bis zu Grössen dritter Ordnung (inclusive) gehenden Genauigkeit dienen sollen. Bei der Berechnung derselben durften in den Ausdrücken von

$$
W_{2}, W_{1} \log B, W_{1} \log (A+B), \frac{M}{\sqrt{\varrho^{2}+z^{2}}}
$$

alle Glieder von höherer als der dritten Ordnung weggelassen werden. So ergiebt sich

$$
\begin{aligned}
& V=\pi a^{2}\left(v_{0}+v_{1} \cos t_{1}+v_{2} \cos 2 t_{1}+v_{3} \cos 3 t_{1}\right) \\
& v_{0}=\beta\left(\log \frac{256}{a^{2}(1+\beta)^{2}}-2\right) \\
& v_{1}=a \beta\left(\frac{-\mathbf{I} \mathbf{I}+9 \beta-\beta^{2}+3 \beta^{3}}{4(\mathbf{I}+\boldsymbol{\beta})}+\log \frac{\mathbf{2}^{6} 6}{a^{2}(\mathbf{I}+\boldsymbol{\beta})^{2}}\right) . \\
& +2 \alpha \frac{\mathrm{I}+\beta-\beta^{2}}{(\mathrm{I}+\beta)^{2}} \cdot \gamma \\
& v_{2}=-\beta_{\mathrm{I}+\beta}^{\mathrm{I}-\beta} \\
& v_{3}=-a \beta \frac{(\mathrm{I}-\beta)(\mathrm{I}+3 \beta)}{6(\mathrm{I}+\beta)^{2}}+a \gamma \cdot \frac{-2+6 \beta+6 \beta^{2}+6 \beta^{3}}{3(\mathrm{I}+\beta)^{3}}
\end{aligned}
$$

Ferner

$$
\begin{aligned}
\frac{M}{\sqrt{\varrho_{1}{ }^{2}+z_{1}^{2}}} & =m_{0}+m_{1} \cos t_{1}+m_{2} \cos 2 t_{1}+m_{3} \cos 3 t_{1} \\
m_{0} & =M \\
m_{1} & =a M\left[1+a^{2}\left(3 / 4-3 / 8 \beta^{2}-1 / 2 \beta \cdot \gamma\right)\right] \\
m_{2} & =a^{2} M^{1 / 4}\left(2+\beta^{2}\right) \\
m_{3} & =a^{3} M\left[1 / \mathrm{s}\left(2+3 \beta^{2}\right)+1 / 2 \beta \gamma\right]
\end{aligned}
$$

Endlich

$1 / 2 n^{2} \varrho^{2}=1 / 2 n^{2}\left(1+1 / 2 a^{2}\right)-n^{2} a \cos t_{1}+1 / 4 n^{2} a^{2} \cos 2 t_{1}$

Die zweite, dritte und vierte der obigen Bedingungsgleichungen

$$
\begin{aligned}
& \pi a^{2} v_{1}+m_{1}-a n^{2}=0 \\
& \pi a^{2} v_{2}+m_{2}+1 / 4 a^{2} n^{2}=0 \\
& \pi a^{2} v_{3}+m_{3}=0
\end{aligned}
$$

gehen dann in die folgenden über

$$
\begin{gathered}
\pi a^{2} \beta\left(\frac{-1 \mathrm{I}+9 \beta-\beta^{2}+3 \beta^{3}}{4(\mathrm{I}+\boldsymbol{\beta})}+\log \frac{25^{6}}{a^{2}(\mathrm{I}+\beta)^{2}}\right)+a^{2} M\left(\frac{3}{4}-\frac{3}{8} \beta^{2}\right)+\left(2 \pi \frac{\mathrm{I}+\beta-\beta^{2}}{(\mathrm{I}+\beta)^{2}}-\frac{1}{2} M \beta\right) a^{2} \gamma+M-n^{2}=0 \\
M\left(\mathrm{2}+\beta^{2}\right)-4 \beta \frac{\mathrm{I}-\beta}{\mathrm{I}+\boldsymbol{\beta}} \pi+n^{2}=0 \\
M^{\frac{2+3^{2}}{8}}-\pi \frac{\beta(\mathrm{I}-\beta)(\mathrm{I}+3 \beta)}{6(\mathrm{I}+\beta)^{2}}+\left(\frac{1}{2} M \beta+\frac{-2+6 \beta+6 \beta^{2}+6 \beta^{3}}{3(\mathrm{I}+\beta)^{3}}\right) \cdot \gamma=0
\end{gathered}
$$


Aus der letzten Gleichung folgt

$$
\gamma=\frac{\mathbf{1}}{4} \frac{3 M\left(2+3 \beta^{2}\right)(\mathbf{I}+\beta)^{2}-4 \pi \beta(\mathbf{I}-\beta)(\mathbf{I}+3 \beta)}{4-\mathbf{I} 2 \beta-12 \beta^{2}-\mathbf{I} 2 \beta^{3}-3 M \beta(\mathbf{I}+\beta)^{3}} \cdot(\mathbf{I}+\beta)
$$

die erste dieser Gleichungen giebt dann

$$
n^{2}=M+a^{2} N
$$

wo

$$
N=\pi \beta\left(\frac{-\mathrm{I} \mathrm{I}+9 \beta-\beta^{2}+3 \beta^{3}}{4(\mathrm{I}+\bar{\beta})}+\log \frac{2^{6}}{\alpha^{2}(\mathrm{I}+\beta)^{2}}\right)+M\left(\frac{3}{4}-\frac{3}{8} \beta^{2}\right)+\left(2 \pi \frac{\mathbf{I}+\beta-\beta^{2}}{(\mathrm{I}+\beta)^{2}}-\frac{\mathbf{I}}{\mathbf{2}} M \beta\right) \cdot \gamma
$$

die zweite Gleichung endlich führt auf die zur Berechnung von $\beta$ dienende

$$
M\left(3+\beta^{2}\right)-\frac{4 \beta(1-\beta)}{1+\beta} \pi+a^{2} N=0
$$

in welcher für die in $N$ vorkommende Grösse $\gamma$ der unter (I) aufgestellte Ausdruck derselben zu setzen ist.

Vernachlässigt man in der vorstehenden Gleichung das mit $a^{2}$ multiplicirte Glied, so ergiebt sich die von Laplace zur Berechnung von $\beta$ gefundene Gleichung

$$
M\left(3+\beta^{2}\right)-\frac{4 \beta(\mathrm{I}-\beta)}{\mathrm{I}+\beta} \pi=0
$$

Die Grösse $\beta$ ist positiv und darf, damit $M$ positiv sei, nicht grösser als $\mathbf{I}$ sein. Daraus folgt, wie Laplace bemerkt hat, dass $\frac{M}{4 \pi}$ eine gewisse Grösse $(0.0543)$ nicht uberschreiten darf. Unter dieser Voraussetzung hat die Laplace'-

sche Gleichung zwei positive Wurzeln. Ist $\beta_{0}$ eine derselben, so erhält man aus (3) bis auf eine Grösse vierter Ordnung

$$
\beta=\beta_{0}-\frac{a^{2} N_{0}}{2 M \beta_{0}-4+\frac{8}{\left(\mathrm{I}+\beta_{0}\right)^{2}}}
$$

wo $N_{0}$ den Werth von $N$ für $\beta_{0}$ bedeutet.

Dieser Werth von $\beta$ in den Ausdruck (I) von $\gamma$ eingesetzt giebt dann auch diese Grösse mit demselben Grade von Annäherung; und ebenso erhält man aus (2)

$$
n^{2}=M+a^{2} N_{0}
$$

Ich bemerke noch, dass, bei hinlänglich kleinem Werthe von $M$, wenn man den kleineren Werth von $\beta$ nimmt, die Krümmung des Querschnittes des Ringes in dem Punkte, der dem Mittelpunkte am nächsten ist, stärker ist, als in dem entgegengesetzten Punkte. Nimmt man den grösseren Werth von $\beta$, so verhält es sich umgekehrt.

Ephemeride des Cometen 1884 Wolf (Fortsetzung zu Nr. 263r).

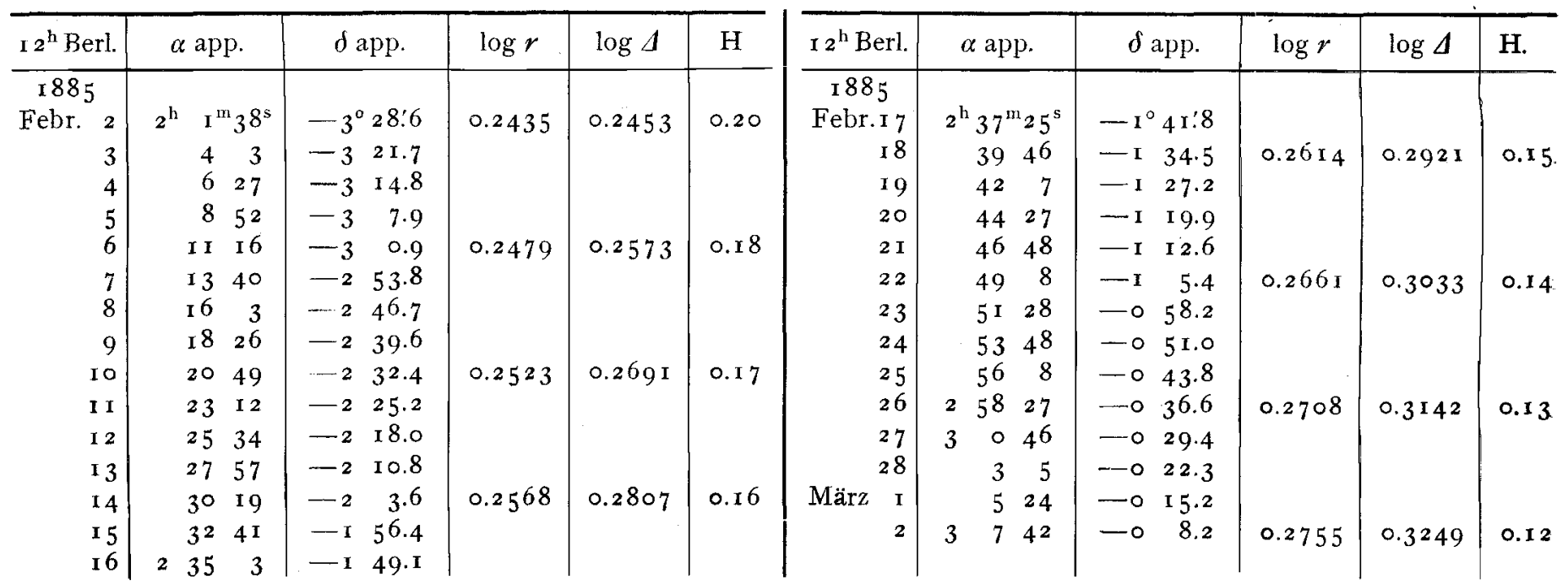

Nach einer Beobachtung des Herrn Tempel in Arcetri von Jan. 5 betrug die Abweichung der Ephemeride $(B-R):+2^{s}$ und -0.1 . Sophie Kowalezesky. Zusätze und Bemerkungen zu Laplace's Untersuchung über die Gestalt der Saturnsringe. 37 . - C. Stechert Ephemeride des Cometen I 884 Wolf. 47. 